Review Article

\title{
A Critical Review on Prosthetic Features Available for Reversed Total Shoulder Arthroplasty
}

\author{
Bart Middernacht, Alexander Van Tongel, and Lieven De Wilde \\ University Hospital, De Pintelaan 185, 9000 Ghent, Belgium \\ Correspondence should be addressed to Alexander Van Tongel; alexander.vantongel@uzgent.be
}

Received 8 June 2016; Revised 24 November 2016; Accepted 27 November 2016

Academic Editor: Gasparini Giulio

Copyright ( 2016 Bart Middernacht et al. This is an open access article distributed under the Creative Commons Attribution License, which permits unrestricted use, distribution, and reproduction in any medium, provided the original work is properly cited.

Reversed total shoulder arthroplasty is a popular treatment in rotator cuff arthropathy and in displaced proximal humeral fractures in elderly. In 2016, 29 models of commercially available designs express this popularity. This study describes all the different design parameters available on the market. Prosthetic differences are found for the baseplate, glenosphere, polyethylene, and humeral component and these differences need to be weighed out carefully for each patient knowing that a gain in one mechanical parameter can balance the loss of another. Patient specific implants may help in the future.

\section{Introduction}

Reversed total shoulder arthroplasty is a very popular treatment in rotator cuff arthropathy and in displaced proximal humeral fractures in elderly patients [1-13]. In 2016, this popularity is expressed by not less than 29 models of commercially available designs (Figure 1).

Although these modern generations of the reverse shoulder prosthesis vary in specific design details, they continue to adhere to Grammont's core principles [14].

In 1987, Grammont et al. [14, 15] introduced two major innovations in the reverse prosthesis: notably a large glenoid hemisphere with no neck and a small almost horizontally inclined (155 degrees) humeral component covering less than half of the hemisphere.

The main biomechanical advantages of this reverse prosthesis according to Grammont's concept are as follows: (1) the large ball offers a greater potential arc of motion and more stability than a small ball, (2) the small lateral offset (absence of neck) places the center of rotation directly in contact with the glenoid surface and reduces the torque at the point of fixation of the glenoid component, (3) medializing the center of rotation recruits more of the deltoid fibers for elevation or abduction, and (4) lowering the humerus increases tension on the deltoid. These biomechanical properties may lead to better functioning of the deltoid by an increase of its lever arm and moment of action, compensating for the lack of a functional rotator cuff [14-16].

However, an important limitation of this nonanatomical prosthesis is its inability to restore active internal and external rotation. This is caused mainly by design limitations of the prosthesis $[17,18]$, producing mechanical impingement and malfunctioning of the rotator cuff remnants [16, 19-21]. It can also be explained by the slackening of the remaining external rotators due to the medialization of the center of rotation [22]. This is why a prosthesis with a built-up lateralized center of rotation exhibits a less weakened external rotation [23, 24]. Another way to overcome this limitation can be found in certain surgical techniques and prosthetic designs creating a more lateral position of the humerus to increase the deltoid wrapping resulting in a better tension in the anterior and posterior fibers $[25,26]$.

A second prosthetic limitation of this reversed principle rises from its hinged rotation (the humeral component rotates around the glenosphere) instead of a spinning rotation (the humeral head rotates on the spot) as seen in an anatomical setting. This type of rotation requires more room, because without it, a conflict between the humeral and glenoid parts can occur. Best known is the scapular notching in which the humerus is in conflict with the infraglenoid tubercle $[16,18,22,27,28]$. This mechanical impingement also exists 


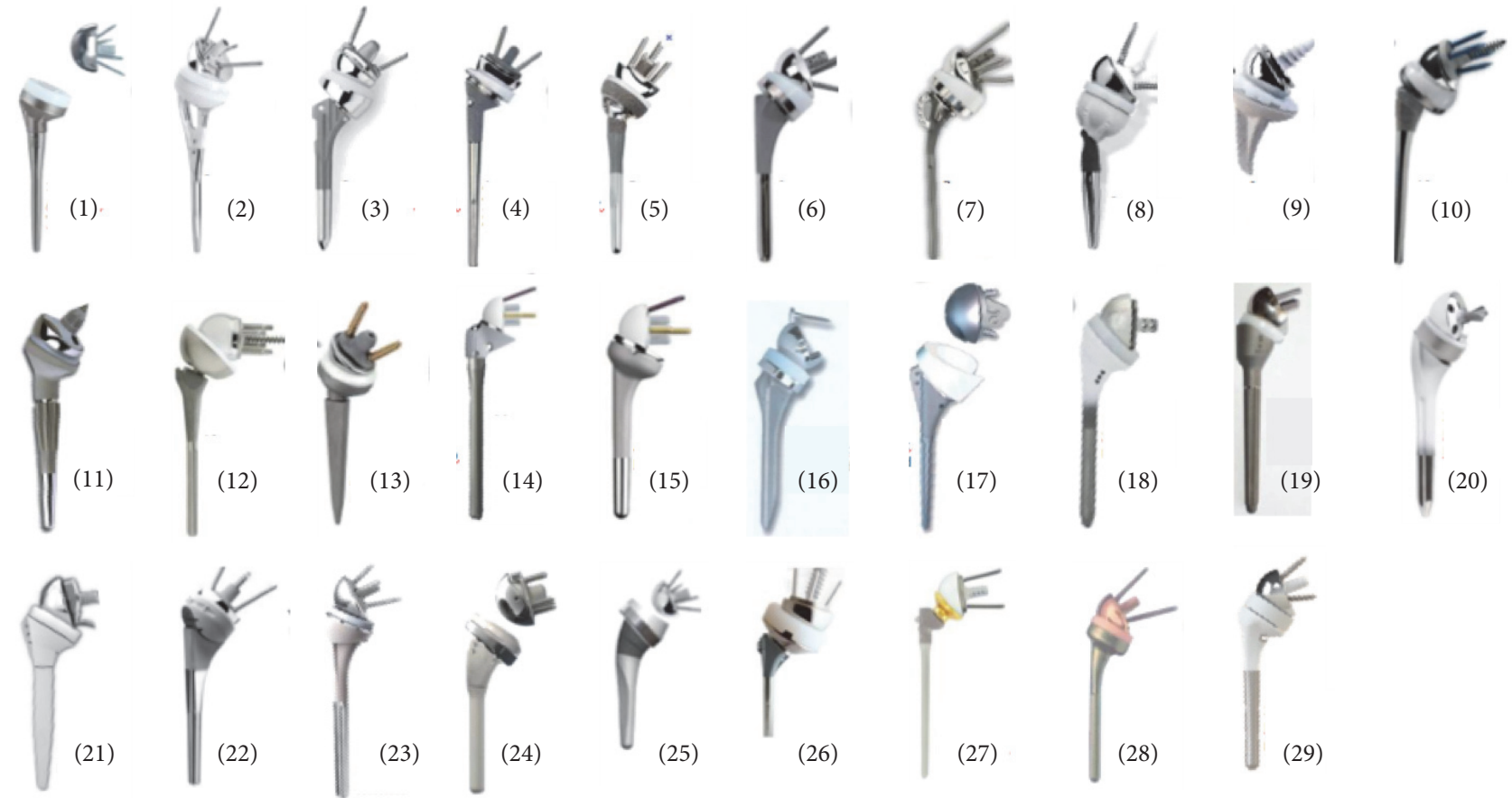

FIGURE 1: 29 models of commercially available reverse prosthesis designs: (1) Aequalis Tornier; (2) Aequalis Fracture Tornier; (3) Anatomical Zimmer; (4) Trabecular Zimmer; (5) Titan Integra; (6) Equinoxe Exactech; (7) Equinoxe Fracture Exactech; (8) TESS Biomet; (9) Verso Biomet;

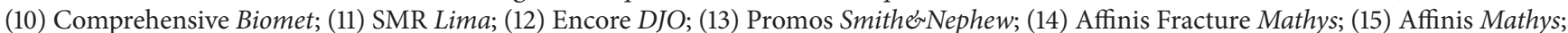
(16) Duocentric Aston Med; (17) Arrow FH; (18) Scultra Euros; (19) NGRi Wright; (20) Unic Evolutis; (21) Géo Biotechni; (22) Univers Arthrex; (23) Delta Xtend Depuy-Synthes; (24) Vaios JRI; (25) Ascend Tornier; (26) Seviin Ingen; (27) Agilon Implantcast; (28) Ulys Ceraver; and (29) Humelock FX Solution.

in the transverse plane of the body, thereby limiting the range of external and internal rotation and possibly leading to mechanical prosthetic failure when the tuberosities bump against the glenoid [17, 29, 30].

The aim of this review is to gather some of the existing literature on the different prosthetic parameters that can influence these biomechanics and kinematics of the reversed shoulder arthroplasty. The differences are to be found at the level of the baseplate, glenosphere, polyethylene, or humeral component.

\section{Baseplate Options}

A baseplate can be oval or circular (Figure 2). For circular baseplate the radius is between $25 \mathrm{~mm}$ and $29 \mathrm{~mm}$ [31, 32]. Oval baseplates are $25 \mathrm{~mm}$ over $34 \mathrm{~mm}$. There even are oval base plates available with an inferior extension placing a protective resurfacing shield over the critical notching part of the scapula [33].

Roche et al. tested, in vitro, the fixation strength of 4 generic baseplates, attached to a low-density polyurethane substrate. They suggested that oval baseplates $(25 \mathrm{~mm} \times$ $34 \mathrm{~mm}$ ) show better fixation characteristics than circular ones $(25 \mathrm{~mm} \times 25 \mathrm{~mm})$ [34]. A round base plate, on the other hand, has the advantage to simplify the surgical technique because a reamer with a smaller diameter can be used and the longitudinal axis of the glenoid does not have to be defined.

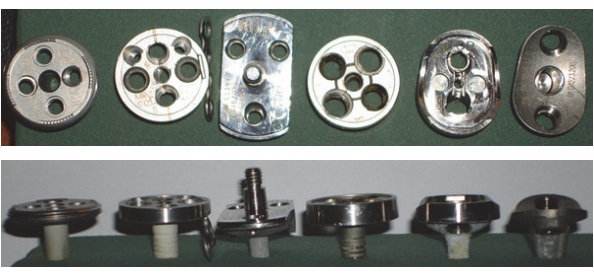

FIGURE 2: Baseplates can ben oval or circular, flat-backed or curvedbacked, and with or without inferior extension.

The backside of the baseplate can be flat or convex (Figure 2) and as a consequence flat and convex reamers can be used to prepare the glenoid. Flat reaming will remove more bone than convex reaming in uniconcave glenoids. In contrast, flat reaming removes less bone than convex reaming in biconcave glenoids. However, this difference is minimal [35]. This fact might be important in osteoporotic bone.

Other differences can be found in terms of preparing the glenoid. There are systems with pin-guided reamers, as well as systems using a nipple-guided technique. A recent study showed that both techniques are equally accurate [35]. Further, a reduced diameter of this reamer would facilitate the reaming process, although scientific evidence is not yet available for this. 


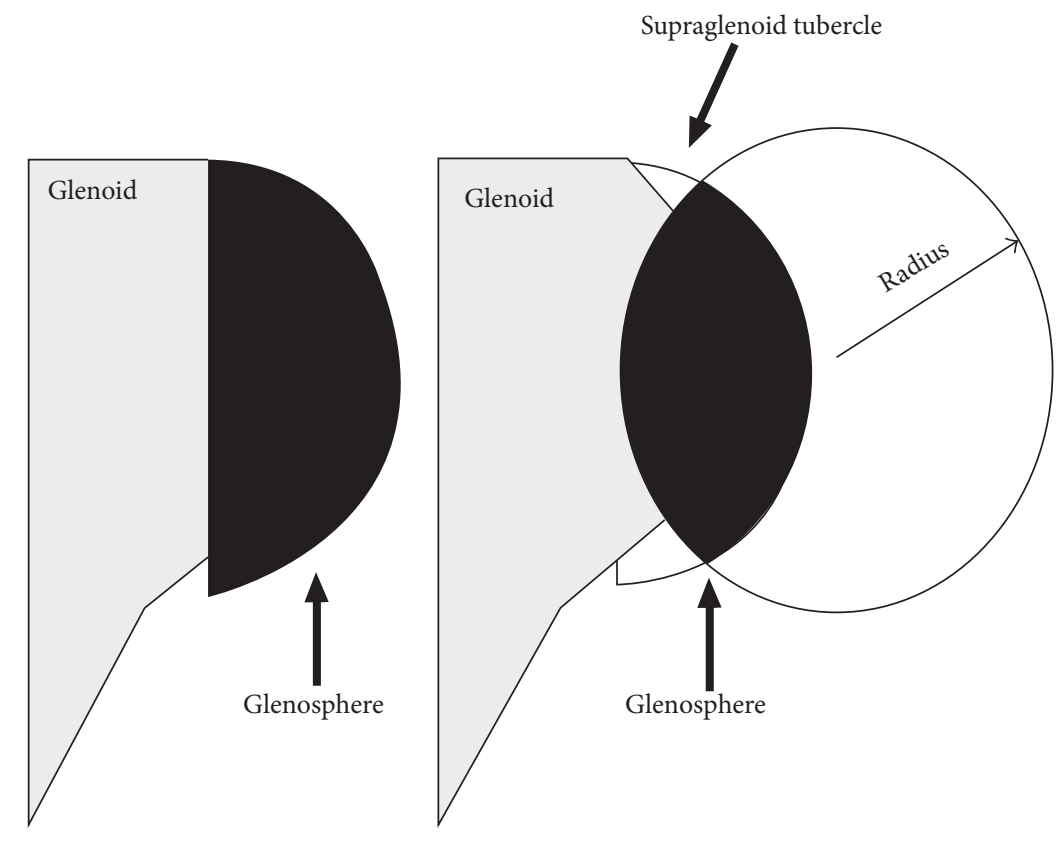

(a)

(b)

Figure 3: The differences in glenosphere placement between a (a) flat base plate and a (b) convex base plate are shown. Both have the same center of rotation [28].

Primary stability can be achieved with a screwed, keeled, single pegged, or double pegged base plate. There is yet no scientific evidence for a difference in stability or fixation between these different types of base plates. However, it can be assumed that baseplates with a central screw have better primary stability than pegged or keeled ones and that the lesser the bone is removed, the lesser the glenoid is weakened.

Regarding the shape of the peg, the literature shows us that a conical peg with hydroxyapatite coating provides the best initial stability [36, 37].

All current baseplates offer the possibility of positioning extra screws for fixation. These screws can be locking or nonlocking screws. There are no studies published that promote the use of variable angle locking screws, although this does seem to be useful for fixation of the baseplate. James et al. investigated the number of screws needed for initial stability [38] and decided that only two locking screws provide sufficient stability and extra screws have no added value. These extra screws become more important when the depth of the central peg anchorage is reduced. If possible, four metaglene screws should be used in these cases of uncontained bone loss to guarantee the highest stability [39].

For secondary stabilization, the coating is responsible for the osseous ingrowth of the base plate. A difference can be made between regrowth of bone around or into the prosthesis.

This latter form can be expected to be more stable but may complicate potential revision surgery in the future.

Finally, there are also augmented baseplates, specially developed for bony defects of the glenoid. These should make it possible to place the baseplate in a correct position, ideally perpendicular to the scapular plane, even in very difficult cases [40].

For these difficult cases, base plates with a long peg are available. If necessary these options can all be combined with bone grafting to overcome bony defects.

It is clear that proper preoperative planning, with determination of the size of the needed augmentation, is necessary.

\section{Glenosphere Options}

Several different morphologies of the glenosphere can be found. The glenosphere may be designed as half a sphere, but it can also be more or less than half this sphere. Being less than half a sphere, it will facilitate the assembly and the surgical procedure. However, a glenosphere of more than half a sphere will allow a lateralization of the center of rotation. This in turn facilitates the function of the remaining rotator cuff $[23,26,41]$. This morphology of the glenosphere determines the lateralization of the center of rotation of the joint. When a convex base plate is used in combination with a half-spherical glenosphere there will be a lateralization of the center of rotation. However, when the aim is to place the center of rotation in the plane of the glenoid, one can combine a convex base plate with a glenosphere that is smaller than half a sphere [28] (Figure 3).

Further, there are also eccentric glenospheres [42] that are placed in a lowered, off centered position (Figure 4). This should reduce the incidence of scapular notching but there will be a greater stress on the baseplate in this position with a higher probability of loosening $[43,44]$. 


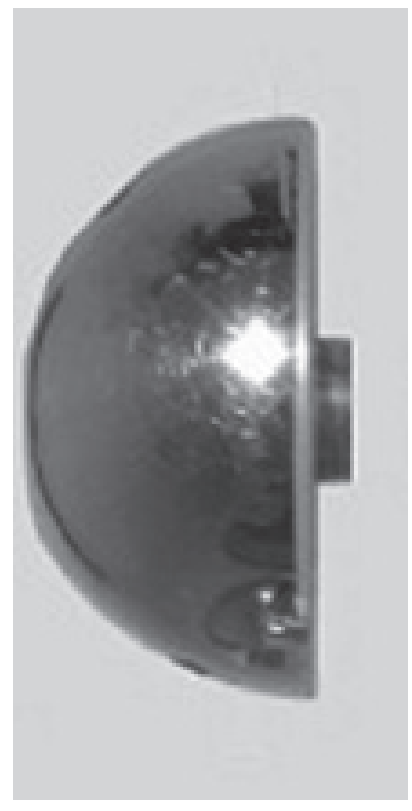

(a)

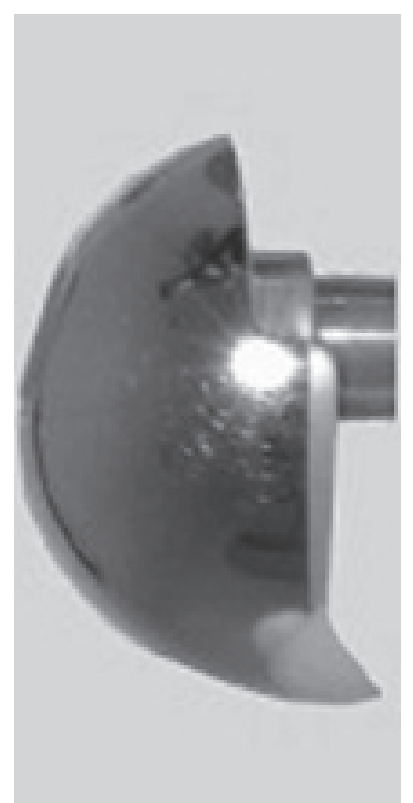

(b)

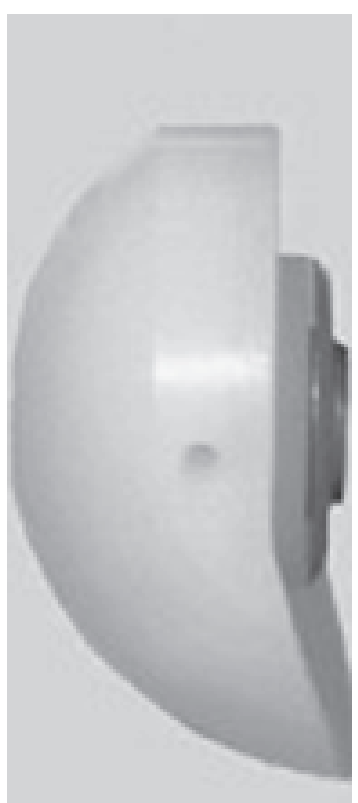

(c)

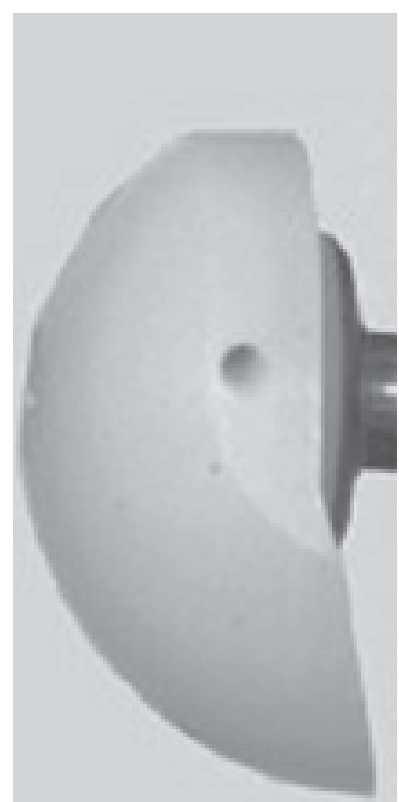

(d)

Figure 4: Different designs of glenospheres: from (a) to (d) $36 \mathrm{~mm}$ concentric, $36 \mathrm{~mm}$ eccentric, $44 \mathrm{~mm}$ polyethylene concentric glenosphere, and $44 \mathrm{~mm}$ polyethylene eccentric glenosphere [43]. Note the inferior extension to prevent impingement.

Finally, glenospheres with an inferior extension are available. This extension could prevent inferior impingement. In a trade-off, it has the disadvantage that more bone needs to be removed for its placement, and, again, the off-axis forces will create greater stress on the baseplate and bigger probability of loosening [43] (Figure 4).

For the size of the glenosphere it has been shown that a larger glenosphere increases the range of motion [45], assuming that a full capsular release was done so no posterior capsule remnant interferes with the internal rotation [46]. A disadvantage of a larger glenosphere is that it complicates the surgery because it is technically more difficult to put in place. Gerber et al. [47] showed in laboratory environment that reverse shoulder prostheses with small glenospheres would be more stable. In vivo, however, stability of the prosthesis is a multifactorial concept. Deltoid function, localization of the center of rotation, stiffness of the soft tissues, and the size of the glenosphere contribute to the stability of the prosthetic joint [47].

Although a biomechanical study shows that increasing the glenosphere diameter significantly increases the joint load and deltoid force [46], the clinical impact of these changes is presently unclear. Finally, it is documented that a larger glenosphere is the best way to prevent inferior scapular notching because of the prosthetic bony overhang [27].

Inferior tilting of the glenosphere is also known to help preventing scapular notching [48-50]. Following this principle, there also exists a glenosphere with a built-in inferior inclination of ten degrees. This configuration should cause less impingement, though this has not yet been proven. This varus-tilt also ensures that less subchondral bone must be removed at the glenoid when a slight varus position is the goal. If a built-in varus-tilt is implanted, the surgeon needs to realize that this lateralizes the center of rotation and thus increases the loads on the glenoid component [25].

There are a few ways to fix the glenosphere onto the baseplate: historically there were bad experiences with threaded glenospheres [16]. Very often loosening was seen after time with this type of fixation. Today most manufacturers have a morse taper. As this connection is made in vivo, it is important to avoid interposition of soft tissues. Disengagement of the glenosphere is, when using the correct technique, fortunately not frequently seen (1.7\%) [51]. The use of a central screw should ease the placement of the glenosphere in the optimal position and can be used without damaging the humeral polyethylene [52].

Regarding the material of the glenosphere the surgeon has two options: all-polyethylene or polished $\mathrm{Cr}-\mathrm{Co}-\mathrm{Ni}$ alloy (Figure 4). Both materials show similar wear, so they are equally valid options [53-55]. A polyethylene glenosphere combined with a metal humeral inlay has the advantage that it creates less polyethylene debris when scapular notching would occur. 55].

Theoretically, this will induce less active osteolysis [53-

\section{Polyethylene Options}

Regarding the polyethylene insert of the humeral component, several variations can be found. It is important to realize that the stability of the prosthesis depends on the amount 


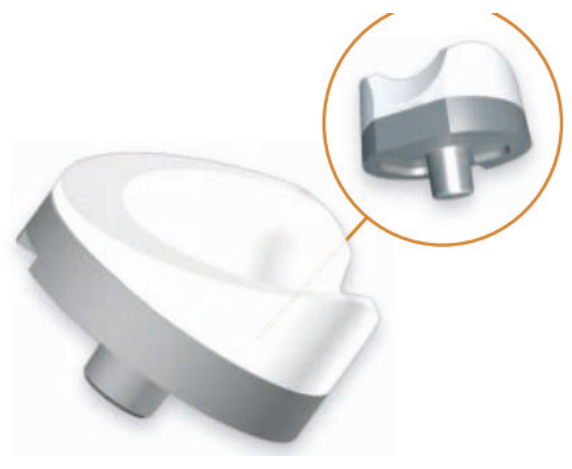

(a)

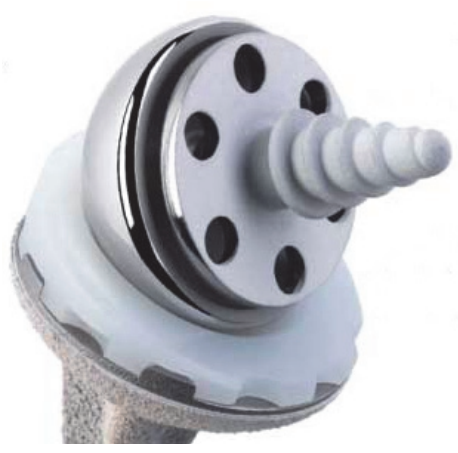

(b)

FIGURE 5: Two types of polyethylene inlay: (a) a horseshoe-shaped inlay [75]; (b) a wedged inlay [76].

of surface of the glenosphere that is in contact with the polyethylene inlay $[47,56]$.

Some companies make a distinction between an inlay with minimal contact surface (high-mobility) and intermediate and maximal contact (constraint, retentive). The latter can be used in the event of instability of the prosthesis. Additional stability afforded by retentive liners should be balanced against the potential for increased wear and the potential for subsequent polyethylene wear-induced aseptic loosening $[57,58]$.

With a smaller contact surface, there will be less stability, but the range of motion will increase. A reduced depth of the inlay also ensures a smaller chance of notching. It has been shown that a reduction of $3 \mathrm{~mm}$ in the depth of the polyethylene inlay results in a gain of range of motion of twelve degrees [27]. A disadvantage of this is, again, the decreased stability of the joint.

The thickness of the insert can also differ to help achieve optimal stability and lateralization but one should be careful not to overstuff the joint creating negative effects on deltoid force and joint loading [26]. There is interadaptibility available so that most sizes of humeral components can be combined with most sizes of glenospheres.

There are also different shapes of inlays on the market. Knowing that inferior rim damage is the predominant cause of polyethylene wear and that this wear can induce mechanical loosening of the glenoid component [59], it seems logical that a horse shoe shaped inlay has an advantage over a circular one (Figure 5), although this has not yet been proven [60]. It is also possible to choose a wedged inlay with a lower medial edge theoretically reducing the likelihood of notching (Figure 5).

\section{Humeral Component Options}

The humeral component has a metaphyseal inclination. This was for the original Grammont prosthesis 155 degrees, instead of the anatomical 135 degrees, and was used in most designs. However, the introduction of the platform systems that allow a conversion from an anatomical to a reverse prosthesis opens up the discussion concerning the optimal inclination.

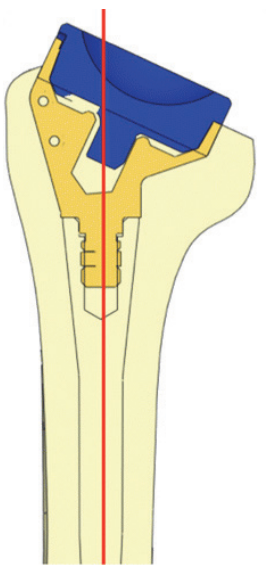

(a)

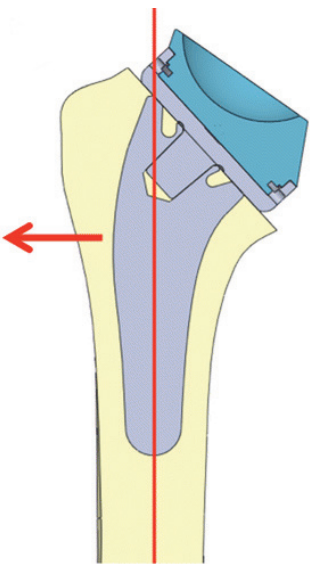

(b)
FIgURE 6: (a) Traditional inlay Grammont RSA with a straight stem and a $155^{\circ}$ inclination. (b) Example of a new design with a curved stem and an onlay humeral tray in a $135^{\circ}$ inclination. The red line passes through the center of the stem. Note that the center of the polyethylene is more medial with the curved stem which results in lateralization of the humerus (red arrow) [62].

A reduction of the inclination to $135^{\circ}$ yields a gain in adduction and reduces the risk of notching. When $135^{\circ}$ is chosen in an onlay-configuration, it also lateralizes the humerus, which optimizes the muscle tension and range of motion [61, 62] (Figure 6). These advantages have to be weighed out to the loss of stability in the initial abduction, which has been confirmed in laboratory conditions by $\mathrm{Oh}$ et al. [63]. For designs with an inclination of 155 degrees, this study describes an increased incidence of scapulohumeral conflict but a higher stability in internal rotation (the most unstable position for anterior stability) [63].

The humeral component can be stemmed, shortstemmed [64, 65], or stemless [66]. The series with stemless prostheses present equal results as the stemmed ones. The right indication is crucial in these cases: in case of primary cuff tear arthropathy, a stemless prosthesis may provide sufficient initial stability. However, for fractures, a stemmed prosthesis will be necessary. In these fracture cases, a 


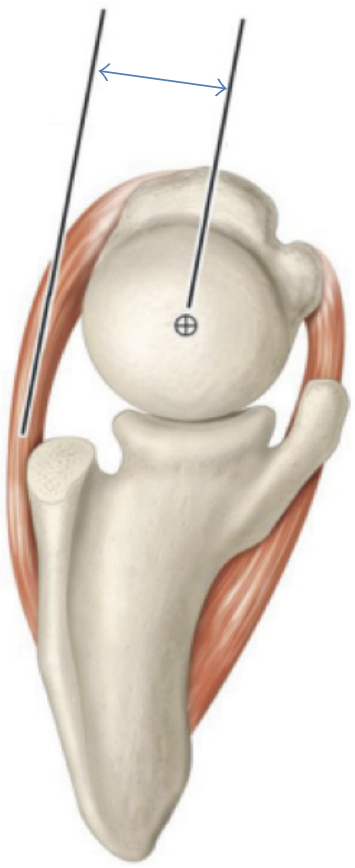

(a)

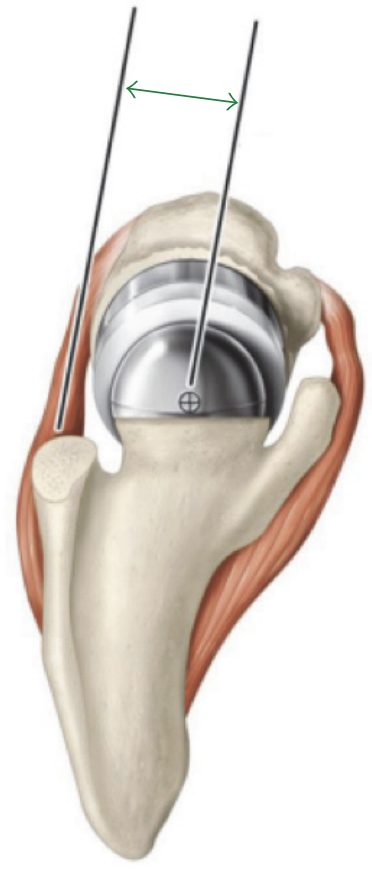

(b)

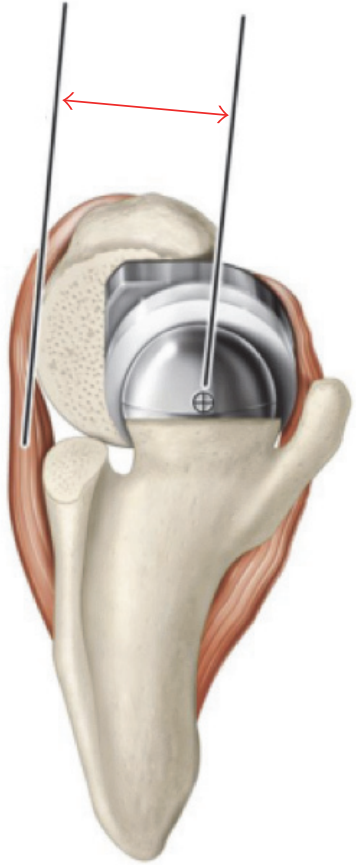

(c)

FIgURE 7: Normal shoulder (a), nonoffset rTSA (b), and posterior-superior offset rTSA (c) [72].

windowed trauma stem is an option and seems to enhance the ingrowth of the tubercles $[67,68]$.

With stemmed prostheses, the surgeon can choose between monoblock and modular humeral types. Monoblocks should prevent dissociation of the prosthetic components in patients where no reconstruction of a viable bony proximal humerus can be expected [61].

Stemmed prostheses can be press-fit or cemented. Both provide similar radiographic and functional outcomes [69] when the press-fit humeral component has a tapered metaphyseal segment. The press-fit cylindrical components have an inferior fixation [70].

In patients where the bone quality cannot provide adequate stability (e.g., fractures, elderly), a cemented prosthesis may be necessary. In case a revision is needed and humeral removal is required, a cemented humeral stem will create more bone loss than can be expected for a press-fit humeral component [71].

At the level of the epiphysis it is possible to place an epiphyseal component with or without posterior offset (Figure 7). Posterior offset allows better anatomical pressfit without anterior bony destruction. This offset also affects the lever arm of the rotator cuff. However, it has not been demonstrated that this effect could have any clinical significance $[72,73]$. Roche et al. have shown that a posterior offset creates a more anatomical tension in the muscles, but the disadvantage is that there can be a subacromial or subcoracoid conflict in extreme rotation or anterior elevation $[72,74]$.

\section{Summary}

Combining some of the available literatures on the different types of design for the reversed total shoulder arthroplasty leads to the following conclusions. Oval baseplate shows better fixation than circular ones but the surgical technique is more demanding. There is no known difference between screwed, keeled, and single or double pegged base plates for primary stabilization. Only two locking screws are needed to provide sufficient stability for the fixation. Several different morphologies of glenospheres are available. The differences have an influence of the center or rotation; a reduction in contact area between the glenosphere surface and the polyethylene increases the mobility but decreases the stability. To overcome the problem of notching, inferior bony overhang seems to be the most effective solution. Prosthetic adaptations are available to assist in avoiding this complication. In the possibility of a future revision, any surgeon needs to differentiate between the advantages and disadvantages of a horizontal or anatomical position of the proximal end of the humeral stem. Medialization and lateralization of the glenoid and humeral part are heavily debated at present and future research will hopefully provide accurate answers.

The orthopaedic surgeon will always have to make some concessions as the ideal balance for different patients may vary several millimeters or degrees. This problem could possibly be resolved in the future, using patient specific implants based on accurate preoperative morphological measurements. 


\section{Competing Interests}

Bart Middernacht has no conflict of interests. Alexander Van Tongel has no conflict of interests. Lieven De Wilde accepts royalties from Depuy-Synthes.

\section{References}

[1] A. Boulahia, T. B. Edwards, G. Walch, and R. V. Baratta, "Early results of a reverse design prosthesis in the treatment of arthritis of the shoulder in elderly patients with a large rotator cuff tear," Orthopedics, vol. 25, no. 2, pp. 129-133, 2002.

[2] L. Laudicina and R. D’Ambrosia, "Management of irreparable rotator cuff tears and glenohumeral arthritis," Orthopedics, vol. 28, no. 4, pp. 382-388, 2005.

[3] M. Frankle, S. Siegal, D. Pupello, A. Saleem, M. Mighell, and M. Vasey, "The reverse shoulder prosthesis for glenohumeral arthritis associated with severe rotator cuff deficiency: a minimum two-year follow-up study of sixty patients," Journal of Bone and Joint Surgery A, vol. 87, no. 8, pp. 1697-1705, 2005.

[4] K. J. Ecklund, T. Q. Lee, J. Tibone, and R. Gupta, "Rotator cuff tear arthropathy," Journal of the American Academy of Orthopaedic Surgeons, vol. 15, no. 6, pp. 340-349, 2007.

[5] P. Boileau, D. Watkinson, A. M. Hatzidakis, and I. Hovorka, "Neer Award 2005: the Grammont reverse shoulder prosthesis: results in cuff tear arthritis, fracture sequelae, and revision arthroplasty," Journal of Shoulder and Elbow Surgery, vol. 15, no. 5, pp. 527-540, 2006.

[6] P. Mulieri, P. Dunning, S. Klein, D. Pupello, and M. Frankle, "Reverse shoulder arthroplasty for the treatment of irreparable rotator cuff tear without glenohumeral arthritis," The Journal of Bone \& Joint Surgery - American Volume, vol. 92, no. 15, pp. 2544-2556, 2010.

[7] G. N. Drake, D. P. O’Connor, and T. B. Edwards, "Indications for reverse total shoulder arthroplasty in rotator cuff disease," Clinical Orthopaedics and Related Research, vol. 468, no. 6, pp. 1526-1533, 2010.

[8] B. M. Nolan, E. Ankerson, and M. J. Wiater, "Reverse total shoulder arthroplasty improves function in cuff tear arthropathy," Clinical Orthopaedics and Related Research, vol. 469, no. 9, pp. 2476-2482, 2011.

[9] E. T. H. Ek, L. Neukom, S. Catanzaro, and C. Gerber, "Reverse total shoulder arthroplasty for massive irreparable rotator cuff tears in patients younger than 65 years old: results after five to fifteen years," Journal of Shoulder and Elbow Surgery, vol. 22, no. 9, pp. 1199-1208, 2013.

[10] M. A. Ramirez, J. Ramirez, and A. M. Murthi, "Reverse total shoulder arthroplasty for irreparable rotator cuff tears and cuff tear arthropathy," Clinics in Sports Medicine, vol. 31, no. 4, pp. 749-759, 2012.

[11] C. D. Jarrett, B. T. Brown, and C. C. Schmidt, "Reverse shoulder arthroplasty," Orthopedic Clinics of North America, vol. 44, no. 3, pp. 389-408, 2013.

[12] S. W. Young, M. Zhu, C. G. Walker, and P. C. Poon, “Comparison of functional outcomes of reverse shoulder arthroplasty with those of hemiarthroplasty in the treatment of cuff-Tear arthropathy a matched-pair analysis," Journal of Bone and Joint Surgery A, vol. 95, no. 10, pp. 910-915, 2013.

[13] W. D. Aumiller and T. M. Kleuser, "Diagnosis and treatment of cuff tear arthropathy," Journal of the American Academy of Physician Assistants, vol. 28, no. 8, pp. 33-38, 2015.
[14] P. M. Grammont and E. Baulot, "The classic: delta shoulder prosthesis for rotator cuff rupture," Clinical Orthopaedics and Related Research, vol. 469, no. 9, p. 2424, 2011.

[15] P. Grammont, P. Trouilloud, J. P. Laffay, and X. Deries, "Etude et réalisation d'une nouvelle prothèse d'épaule," Rhumatologie, vol. 39, no. 10, pp. 407-418, 1987.

[16] P. Boileau, D. J. Watkinson, A. M. Hatzidakis, and F. Balg, "Grammont reverse prosthesis: design, rationale, and biomechanics," Journal of Shoulder and Elbow Surgery, vol. 14, no. 1, pp. S147-S161, 2005.

[17] L. De Wilde and G. Walch, "Humeral prosthetic failure of reversed total shoulder arthroplasty: a report of three cases," Journal of Shoulder and Elbow Surgery, vol. 15, no. 2, pp. 260264, 2006.

[18] R. W. Nyffeler, C. M. L. Werner, B. R. Simmen, and C. Gerber, "Analysis of a retrieved Delta III total shoulder prosthesis," Journal of Bone and Joint Surgery B, vol. 86, no. 8, pp. 1187-1191, 2004.

[19] L. Seebauer, W. Walter, and W. W. Keyl, "Reverse total shoulder arthroplasty for the treatment of defect arthropathy," Operative Orthopädie und Traumatologie, vol. 17, no. 1, pp. 1-24, 2005.

[20] C. M. L. Werner, P. A. Steinmann, M. Gilbart, and C. Gerber, "Treatment of painful pseudoparesis due to irreparable rotator cuff dysfunction with the Delta III reverse-ball-and-socket total shoulder prosthesis," Journal of Bone and Joint Surgery A, vol. 87, no. 7, pp. 1476-1486, 2005.

[21] F. Sirveaux, L. Favard, D. Oudet, D. Huquet, G. Walch, and D. Molé, "Grammont inverted total shoulder arthroplasty in the treatment of glenohumeral osteoarthritis with massive rupture of the cuff. Results of a multicentre study of 80 shoulders," Journal of Bone and Joint Surgery - Series B, vol. 86, no. 3, pp. 388-395, 2004.

[22] C. Delloye, D. Joris, A. Colette, A. Eudier, and J.-E. Dubuc, "Mechanical complications of total shoulder inverted prosthesis," Revue de Chirurgie Orthopedique et Reparatrice de l'Appareil Moteur, vol. 88, no. 4, pp. 410-414, 2002.

[23] S. Greiner, C. Schmidt, C. König, C. Perka, and S. Herrmann, "Lateralized reverse shoulder arthroplasty maintains rotational function of the remaining rotator cuff," Clinical Orthopaedics and Related Research, vol. 471, no. 3, pp. 940-946, 2013.

[24] M. A. Hamilton, C. P. Roche, P. Diep, P.-H. Flurin, and H. D. Routman, "Effect of prosthesis design on muscle length and moment arms in reverse total shoulder arthroplasty," Bulletin of the NYU Hospital for Joint Diseases, vol. 71, supplement 2, pp. S31-S35, 2013.

[25] C. P. Roche, P. Diep, M. Hamilton et al., "Impact of inferior glenoid tilt, humeral retroversion, bone grafting, and design parameters on muscle length and deltoid wrapping in reverse shoulder arthroplasty," Bulletin of the NYU Hospital for Joint Diseases, vol. 71, no. 4, pp. 284-293, 2013.

[26] J. W. Giles, G. D. G. Langohr, J. A. Johnson, and G. S. Athwal, "Implant design variations in reverse total shoulder arthroplasty influence the required deltoid force and resultant joint load," Clinical Orthopaedics and Related Research, vol. 473, no. 11, pp. 3615-3626, 2015.

[27] L. F. De Wilde, D. Poncet, B. Middernacht, and A. Ekelund, "Prosthetic overhang is the most effective way to prevent scapular conflict in a reverse total shoulder prosthesis," Acta Orthopaedica, vol. 81, no. 6, pp. 719-726, 2010.

[28] B. Middernacht, P.-J. De Roo, G. Van Maele, and L. F. De Wilde, "Consequences of scapular anatomy for reversed total shoulder 
arthroplasty," Clinical Orthopaedics and Related Research, vol. 466, no. 6, pp. 1410-1418, 2008.

[29] P. Boileau, G. Moineau, Y. Roussanne, and K. O’Shea, “Bony increased-offset reversed shoulder arthroplasty: minimizing scapular impingement while maximizing glenoid fixation," Clinical Orthopaedics and Related Research, vol. 469, no. 9, pp. 2558-2567, 2011.

[30] D. R. Stephenson, J. H. Oh, M. H. McGarry, G. F. Rick Hatch, and T. Q. Lee, "Effect of humeral component version on impingement in reverse total shoulder arthroplasty," Journal of Shoulder and Elbow Surgery, vol. 20, no. 4, pp. 652-658, 2011.

[31] S.-W. Chae, S.-Y. Kim, H. Lee, J.-R. Yon, J. Lee, and S.-H. Han, "Effect of baseplate size on primary glenoid stability and impingement-free range of motion in reverse shoulder arthroplasty," BMC Musculoskeletal Disorders, vol. 15, no. 1, article 417, 2014.

[32] G. S. Athwal and K. J. Faber, "Outcomes of reverse shoulder arthroplasty using a mini $25-\mathrm{mm}$ glenoid baseplate," International Orthopaedics, vol. 40, no. 1, pp. 109-113, 2016.

[33] P. Trouilloud, M. Gonzalvez, P. Martz et al., "Duocentric ${ }^{\circledR}$ reversed shoulder prosthesis and Personal Fit ${ }^{\circledR}$ templates: innovative strategies to optimize prosthesis positioning and prevent scapular notching," European Journal of Orthopaedic Surgery \& Traumatology, vol. 24, no. 4, pp. 483-495, 2014.

[34] C. P. Roche, N. J. Stroud, P.-H. Flurin, T. W. Wright, J. D. Zuckerman, and M. J. DiPaola, "Reverse shoulder glenoid baseplate fixation: a comparison of flat-back versus curved-back designs and oval versus circular designs with 2 different offset glenospheres," Journal of Shoulder and Elbow Surgery, vol. 23, no. 9, pp. 1388-1394, 2014.

[35] A. Karelse, S. Leuridan, A. Van Tongel, I. M. Piepers, P. Debeer, and L. F. De Wilde, "A glenoid reaming study: how accurate are current reaming techniques?" Journal of Shoulder and Elbow Surgery, vol. 23, no. 8, pp. 1120-1127, 2014.

[36] A. R. Hopkins and U. N. Hansen, "Primary stability in reversedanatomy glenoid components," Proceedings of the Institution of Mechanical Engineers, Part H: Journal of Engineering in Medicine, vol. 223, no. 7, pp. 805-812, 2009.

[37] S. C. Mordecai, S. M. Lambert, J. M. Meswania, G. W. Blunn, I. L. Bayley, and S. J. G. Taylor, "An experimental glenoid rim strain analysis for an improved reverse anatomy shoulder implant fixation," Journal of Orthopaedic Research, vol. 30, no. 6, pp. 998-1003, 2012.

[38] J. James, M. A. Allison, F. W. Werner et al., "Reverse shoulder arthroplasty glenoid fixation: is there a benefit in using four instead of two screws?" Journal of Shoulder and Elbow Surgery, vol. 22, no. 8, pp. 1030-1036, 2013.

[39] M. Königshausen, B. Jettkant, N. Sverdlova et al., "Influence of different peg length in glenoid bone loss: a biomechanical analysis regarding primary stability of the glenoid baseplate in reverse shoulder arthroplasty," Technology and Health Care, vol. 23, no. 6, pp. 855-869, 2015.

[40] G. J. Gilot, "Addressing glenoid erosion in reverse total shoulder arthroplasty," Bulletin of the Hospital for Joint Diseases, vol. 71, pp. S51-S53, 2013.

[41] H. R. Hoenecke, C. Flores-Hernandez, and D. D. D'Lima, "Reverse total shoulder arthroplasty component center of rotation affects muscle function," Journal of Shoulder and Elbow Surgery, vol. 23, no. 8, pp. 1128-1135, 2014.

[42] P. C. Poon, J. Chou, S. W. Young, and T. Astley, "A comparison of concentric and eccentric glenospheres in reverse shoulder arthroplasty: a randomized controlled trial," Journal of Bone and Joint Surgery-American Volume, vol. 96, no. 16, pp. e138.1e138.7, 2014.

[43] J. Chou, S. F. Malak, I. A. Anderson, T. Astley, and P. C. Poon, "Biomechanical evaluation of different designs of glenospheres in the SMR reverse total shoulder prosthesis: range of motion and risk of scapular notching," Journal of Shoulder and Elbow Surgery, vol. 18, no. 3, pp. 354-359, 2009.

[44] C.-C. Yang, C.-L. Lu, C.-H. Wu et al., "Stress analysis of glenoid component in design of reverse shoulder prosthesis using finite element method," Journal of Shoulder and Elbow Surgery, vol. 22, no. 7, pp. 932-939, 2013.

[45] J. Berhouet, P. Garaud, and L. Favard, "Influence of glenoid component design and humeral component retroversion on internal and external rotation in reverse shoulder arthroplasty: a cadaver study," Orthopaedics \& Traumatology: Surgery \& Research, vol. 99, no. 8, pp. 887-894, 2013.

[46] G. D. G. Langohr, J. W. Giles, G. S. Athwal, and J. A. Johnson, "The effect of glenosphere diameter in reverse shoulder arthroplasty on muscle force, joint load, and range of motion," Journal of Shoulder and Elbow Surgery, vol. 24, no. 6, pp. 972-979, 2015.

[47] C. Gerber, S. D. Pennington, and R. W. Nyffeler, "Reverse total shoulder arthroplasty," Journal of the American Academy of Orthopaedic Surgeons, vol. 17, no. 5, pp. 284-295, 2009.

[48] C. Lévigne, J. Garret, P. Boileau, G. Alami, L. Favard, and G. Walch, "Scapular notching in reverse shoulder arthroplasty: is it important to avoid it and how?" Clinical Orthopaedics and Related Research, vol. 469, no. 9, pp. 2512-2520, 2011.

[49] R. W. Nyffeler, C. M. L. Werner, and C. Gerber, "Biomechanical relevance of glenoid component positioning in the reverse Delta III total shoulder prosthesis," Journal of Shoulder and Elbow Surgery, vol. 14, no. 5, pp. 524-528, 2005.

[50] S. Gutiérrez, J. C. Levy, M. A. Frankle et al., "Evaluation of abduction range of motion and avoidance of inferior scapular impingement in a reverse shoulder model," Journal of Shoulder and Elbow Surgery, vol. 17, no. 4, pp. 608-615, 2008.

[51] B. Middernacht, L. De Wilde, D. Molé, L. Favard, and P. Debeer, "Glenosphere disengagement: a potentially serious default in reverse shoulder surgery," Clinical Orthopaedics and Related Research, vol. 466, no. 4, pp. 892-898, 2008.

[52] Z. M. Vaupel, K. C. Baker, M. D. Kurdziel, and J. M. Wiater, "Wear simulation of reverse total shoulder arthroplasty systems: effect of glenosphere design," Journal of Shoulder and Elbow Surgery, vol. 21, no. 10, pp. 1422-1429, 2012.

[53] G. Kohut, F. Dallmann, and U. Irlenbusch, "Wear-induced loss of mass in reversed total shoulder arthroplasty with conventional and inverted bearing materials," Journal of Biomechanics, vol. 45, no. 3, pp. 469-473, 2012.

[54] U. Irlenbusch and G. Kohut, "Evaluation of a new baseplate in reverse total shoulder arthroplasty-comparison of biomechanical testing of stability with roentgenological follow up criteria," Orthopaedics and Traumatology: Surgery and Research, vol. 101, no. 2, pp. 185-190, 2015.

[55] U. Irlenbusch, M. J. Kääb, G. Kohut, J. Proust, F. Reuther, and T. Joudet, "Reversed shoulder arthroplasty with inversed bearing materials: 2-year clinical and radiographic results in 101 patients," Archives of Orthopaedic and Trauma Surgery, vol. 135, no. 2, pp. 161-169, 2015.

[56] S. Gutiérrez, Z.-P. Luo, J. Levy, and M. A. Frankle, "Arc of motion and socket depth in reverse shoulder implants," Clinical Biomechanics, vol. 24, no. 6, pp. 473-479, 2009. 
[57] S. Carpenter, D. Pinkas, M. D. Newton, M. D. Kurdziel, K. C. Baker, and J. M. Wiater, "Wear rates of retentive versus nonretentive reverse total shoulder arthroplasty liners in an in vitro wear simulation," Journal of Shoulder and Elbow Surgery, vol. 24, no. 9, pp. 1372-1379, 2015.

[58] T. Smith, A. Bäunker, M. Krämer et al., "Biomechanical evaluation of inferior scapula notching of reverse shoulder arthroplasty depending on implant configuration and scapula neck anatomy," International Journal of Shoulder Surgery, vol. 9, no. 4, pp. 103-109, 2015.

[59] J. S. Day, D. W. MacDonald, M. Olsen, C. Getz, G. R. Williams, and S. M. Kurtz, "Polyethylene wear in retrieved reverse total shoulder components," Journal of Shoulder and Elbow Surgery, vol. 21, no. 5, pp. 667-674, 2012.

[60] A. Lädermann, B. Gueorguiev, C. Charbonnier et al., "Scapular notching on kinematic simulated range of motion after reverse shoulder arthroplasty is not the result of impingement in adduction," Medicine (Baltimore), vol. 94, no. 38, Article ID $\mathrm{e} 1615,2015$.

[61] B.-K. Jeon, K. A. Panchal, J.-H. Ji et al., "Combined effect of change in humeral neck-shaft angle and retroversion on shoulder range of motion in reverse total shoulder arthroplasty-a simulation study," Clinical Biomechanics, vol. 31, pp. 12-19, 2016.

[62] A. Lädermann, P. J. Denard, P. Boileau et al., "Effect of humeral stem design on humeral position and range of motion in reverse shoulder arthroplasty," International Orthopaedics, vol. 39, no. 11, pp. 2205-2213, 2015.

[63] J. H. Oh, S.-J. Shin, M. H. McGarry, J. H. Scott, N. Heckmann, and T. Q. Lee, "Biomechanical effects of humeral neck-shaft angle and subscapularis integrity in reverse total shoulder arthroplasty," Journal of Shoulder and Elbow Surgery, vol. 23, no. 8, pp. 1091-1098, 2014.

[64] E. Atoun, A. Van Tongel, N. Hous et al., "Reverse shoulder arthroplasty with a short metaphyseal humeral stem," International Orthopaedics, vol. 38, no. 6, pp. 1213-1218, 2014.

[65] S. A. Giuseffi, P. Streubel, J. Sperling, and J. Sanchez-Sotelo, "Short-stem uncemented primary reverse shoulder arthroplasty: clinical and radiological outcomes," Bone and Joint Journal, vol. 96, no. 4, pp. 526-529, 2014.

[66] R. Ballas and L. Béguin, "Results of a stemless reverse shoulder prosthesis at more than 58 months mean without loosening," Journal of Shoulder and Elbow Surgery, vol. 22, no. 9, pp. e1-e6, 2013.

[67] F. Reuther, G. Kohut, and S. Nijs, "Newly developed modular reverse fracture endoprosthesis in non-reconstructable humeral head fracture in old people," Operative Orthopadie und Traumatologie, vol. 26, no. 4, pp. 369-382, 2014.

[68] M. Ross, B. Hope, A. Stokes, S. E. Peters, I. McLeod, and P. F. R. Duke, "Reverse shoulder arthroplasty for the treatment of threepart and four-part proximal humeral fractures in the elderly," Journal of Shoulder and Elbow Surgery, vol. 24, no. 2, pp. 215222, 2015.

[69] J. J. King, K. W. Farmer, A. M. Struk, and T. W. Wright, "Uncemented versus cemented humeral stem fixation in reverse shoulder arthroplasty," International Orthopaedics, vol. 39, no. 2, pp. 291-298, 2015.

[70] F. A. Matsen III, J. P. Iannotti, and C. A. Rockwood Jr., "Humeral fixation by press-fitting of a tapered metaphyseal stem. A prospective radiographic study," Journal of Bone and Joint Surgery A, vol. 85, no. 2, pp. 304-308, 2003.

[71] J. A. Zavala, J. C. Clark, M. J. Kissenberth, S. J. Tolan, and R. J. Hawkins, "Management of deep infection after reverse total shoulder arthroplasty: a case series," Journal of Shoulder and Elbow Surgery, vol. 21, no. 10, pp. 1310-1315, 2012.

[72] C. P. Roche, M. A. Hamilton, P. Diep, P.-H. Flurin, and H. D. Routman, "Design rationale for a posterior/superior offset reverse shoulder prosthesis," Bulletin of the NYU Hospital for Joint Diseases, vol. 71, no. 2, pp. S18-S24, 2013.

[73] J. Berhouet, A. Kontaxis, L. V. Gulotta et al., "Effects of the humeral tray component positioning for onlay reverse shoulder arthroplasty design: a biomechanical analysis," Journal of Shoulder and Elbow Surgery, vol. 24, no. 4, pp. 569-577, 2015.

[74] N. J. Dedy, M. Stangenberg, D. Liem et al., "Effect of posterior offset humeral components on range of motion in reverse shoulder arthroplasty," International Orthopaedics, vol. 35, no. 4, pp. 549-554, 2011.

[75] FH ORTHO Simply. Better, "FH Ortho," http://www.fhortho .com/.

[76] Innovative Design Orthopaedics: 'Innovative Design Orthopaedics: -Verso', http://www.idorth.com/productverso/. 


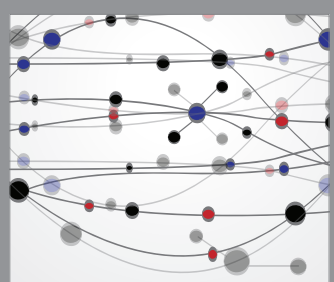

The Scientific World Journal
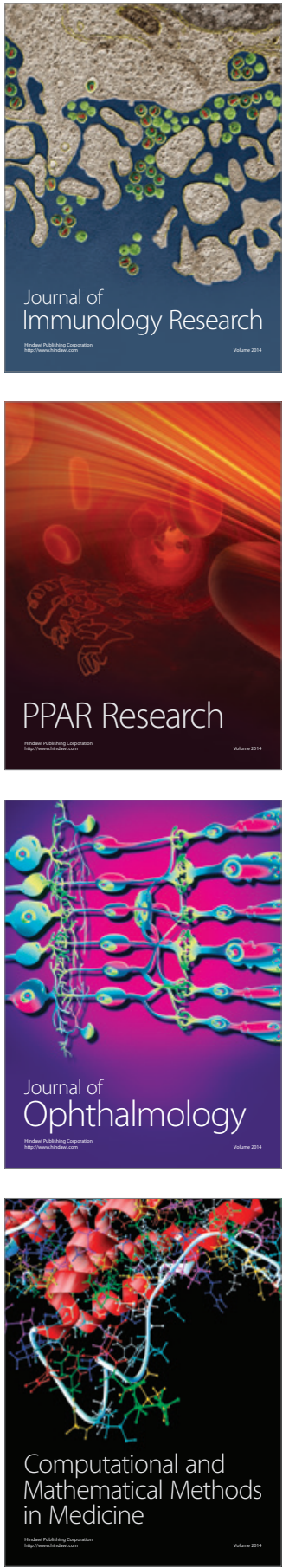

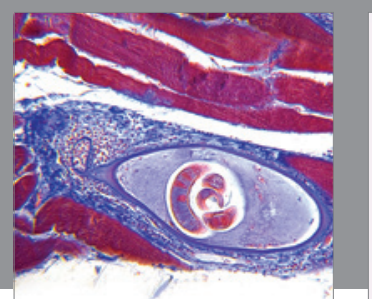

Gastroenterology Research and Practice

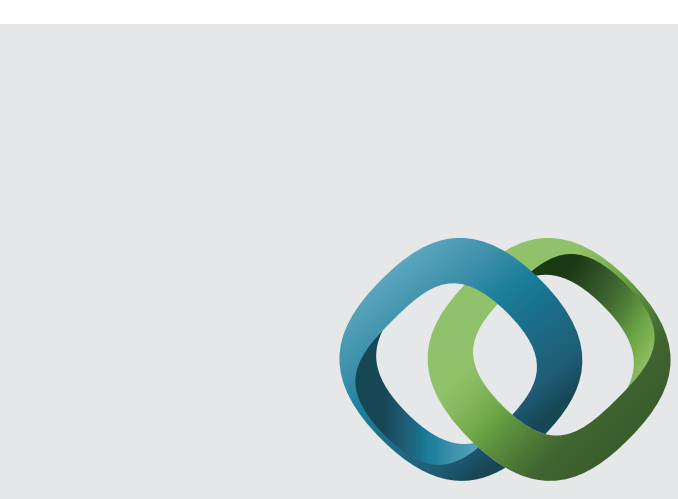

\section{Hindawi}

Submit your manuscripts at

http://www.hindawi.com
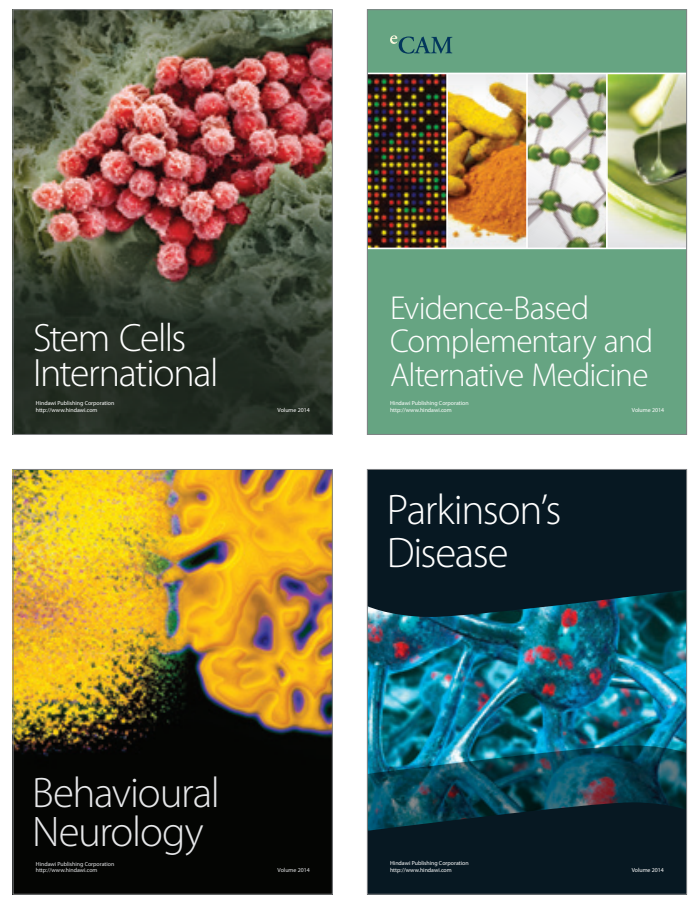
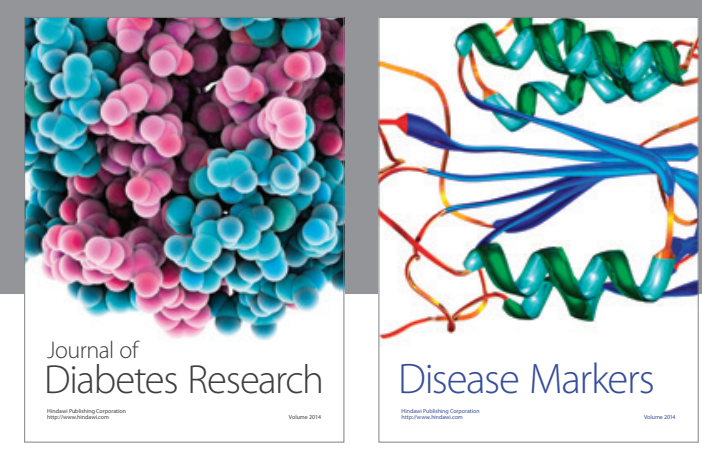

Disease Markers
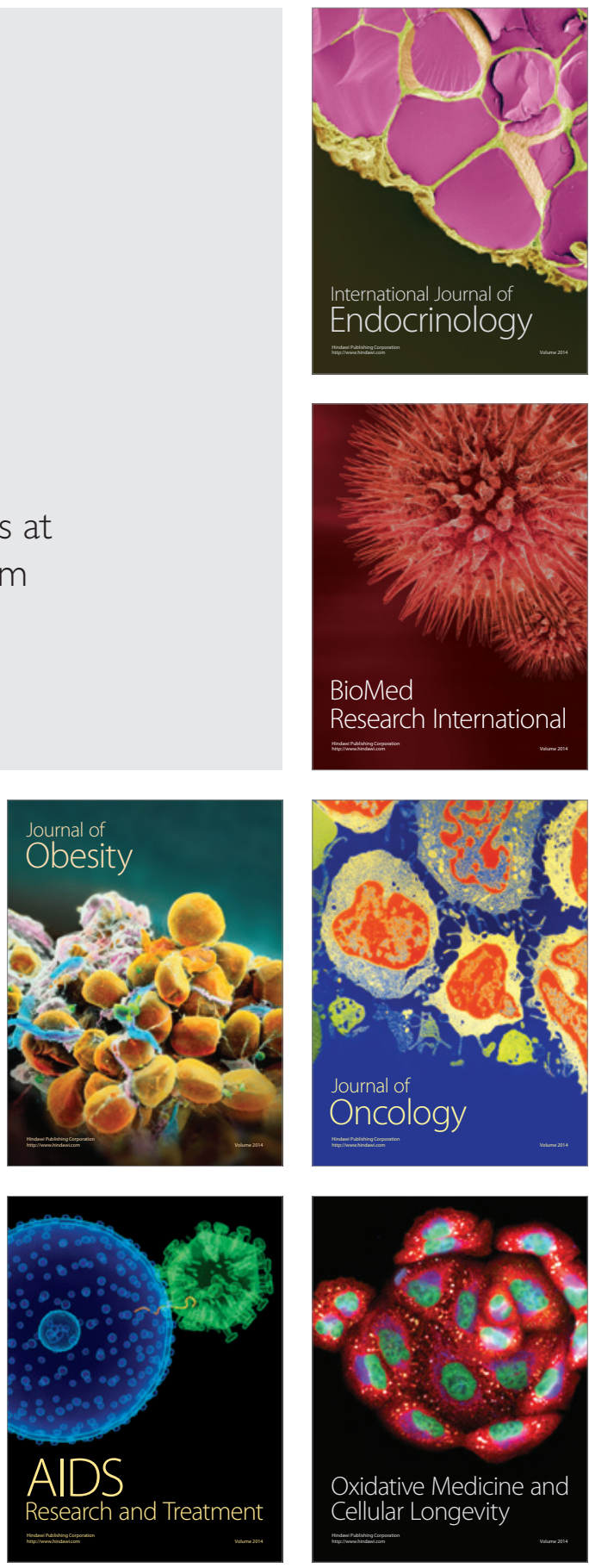